ournal of Fractional Calculus and Nonlinear Systems J Frac Calc \& Nonlinear Sys

\title{
Some Ostrowski Type Integral Inequalities using Hypergeometric Functions
}

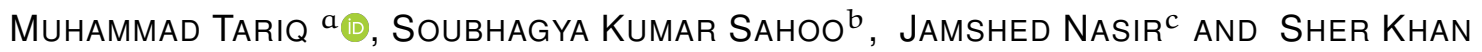 \\ AWAN $^{\mathrm{a}}$ \\ a Mehran University of Engineering and Technology, Jamshoro, Pakistan. \\ b Siksha O Anusandhan University, Bhubaneswar, Odisha, India. \\ c Virtual University Islamabad, Lahore Campus, Pakistan.
}

• Received: 20.03.2021 • Accepted: 23.06.2021 • Published Online: 29.06.2021

\begin{abstract}
The main objective of this paper is basically to acquire some new extensions of Ostrowski type inequalities for the function whose first derivatives absolute value are s-type $p$-convex. We initially presented a new auxiliary definition namely s-type $p$-convex function. Some beautiful algebraic properties and examples related to the newly introduced definition are discussed. We additionally investigated some beautiful cases that can be derived from the novel refinements of the paper. These new results yield us some generalizations of the prior results. We trust that the techniques introduced in this paper will further motivate intrigued researchers.
\end{abstract}

Keywords: Convex function, $\mathrm{p}$-convex function, s-type convex function, s-type p-convex function. 2010 MSC: 26A51, 26A33, 26D07, 26D10, $26 \mathrm{D} 15$.

\section{Introduction}

The theory of convexity is an amazing and compelling methodology for contemplating enormous beautiful issues that emerge in different fields of pure and applied sciences. Numerous new structures have been presented and researched involving convex sets and related functions. This theory has a rich history and has been the concentration and inspiration for uncommon investigation for more than a century in mathematical science. Numerous speculations, variations, and theories of the convexity theory have pulled in the thought of various mathematicians. In the history of advancement calculus, integral inequalities have played a key role in the theory of differential and integral equations. The study of various types of integral inequalities like Ostrowski inequality, Hermite-Hadamard inequality, Simpson inequality etc, has been the focus of great attention for over a century by a number of mathematicians.

\footnotetext{
*Corresponding author: captaintariq2187@gmail.com
} 
A few mathematicians had determined some new variations related to convex functions, see, for instance, $[1,2,3,4]$ and the references therein. This theory had not only interesting and deep results in different branches of engineering and mathematical sciences, but due to widespread view and have a lot of applications, this theory provides numerical quadrature and amazing tools for researchers to tackle and to solve a wide class of related and unrelated problems. Besides, it is marvelous that convexity offers to multiply thoughts and rich applications in each part of pure and applied science. Hermite-Hadamard demonstrated an integral inequality including functions that give us a necessary condition for a function to be convex in nature.

The theory of convexity moreover accepted a critical part in the progression of the idea of inequalities. In different fields of research, inequalities have a great deal of uses in optimization problems, statistical problems, financial issues, numerical analysis problems, probability theory, etc. Inequalities have an intriguing mathematical model because of its significant applications in traditional calculus, fractional calculus, quantum calculus, interval calculus, stochastic, time scale calculus, fractal sets, etc. For the applications, we encourage the readers to go through $[5,6,7,8]$. In such a manner, Ostrowski type inequality is quite possibly one of the most prominent contemplated results.

In literature the well known Ostrowski inequality was first coined in 1938 (see[9]) and is defined as:

Let $\psi: \mathbb{A} \subseteq \mathbb{R} \rightarrow \mathbb{R}$ be a differentiable mapping on $\mathbb{A}^{\circ}$, the interior of the interval $\mathbb{A}$, such that $\psi \in \mathrm{L}\left[\mu_{1}, \mu_{2}\right]$, where $\mu_{1}, \mu_{2} \in \mathbb{A}$ with $\mu_{2}>\mu_{1}$. If $\left|\psi^{\prime}(z)\right| \leqslant K$, for all $z \in\left[\mu_{1}, \mu_{2}\right]$, then the following inequality holds:

$$
\left|\psi(z)-\frac{1}{\mu_{2}-\mu_{1}} \int_{\mu_{1}}^{\mu_{2}} \psi(u) d u\right| \leqslant K\left(\mu_{2}-\mu_{1}\right)\left[\frac{1}{4}+\frac{\left(z-\frac{\mu_{1}+\mu_{2}}{2}\right)^{2}}{\left(\mu_{2}-\mu_{1}\right)^{2}}\right] .
$$

This inequality has wide applications in the field of probability, mathematical analysis and numerical analysis. Recently, this inequality has been extended and generalized using different methodologies and various convex type functions. For some recent result and their related generalizations regarding Ostrowski inequality (see $[10,11,12,13,14]$ ). This inequality presents an upper bound for the approximation of the integral average $\frac{1}{\mu_{2}-\mu_{1}} \int_{\mu_{1}}^{\mu_{2}} \psi(u) d u$ by the value of $\mu(u)$ at the point $u \in\left[\mu_{1}, \mu_{2}\right]$.

\section{Preliminaries}

Definition 2.1. [15] A function $\psi: \mathbb{A}=\left[\mu_{1}, \mu_{2}\right] \rightarrow \mathbb{R}$ is called convex, if

$$
\psi\left(\omega \mu_{1}+(1-\omega) \mu_{2}\right) \leqslant \omega \psi\left(\mu_{1}\right)+(1-\omega) \psi\left(\mu_{2}\right)
$$

holds for all $\mu_{1}, \mu_{2} \in \mathbb{A}$ and $\omega \in[0,1]$.

If the above inequality reverses, then $\psi$ is said to be concave.

The Hermite-Hadamard inequality plays an amazing and magnificent role in the literature, no one can refuse its significance and importance. This type of inequality has 
remained an area of great interest due to its widespread view and applications in the field of mathematical analysis. If $\psi: \mathbb{A} \subset \mathbb{R} \rightarrow \mathbb{R}$ is convex function for $\mu_{1}, \mu_{2} \in \mathbb{A}$ and $\mu_{1}<\mu_{2}$, then

$$
\psi\left(\frac{\mu_{1}+\mu_{2}}{2}\right) \leqslant \frac{1}{\mu_{2}-\mu_{1}} \int_{\mu_{1}}^{\mu_{2}} \psi(\omega) \mathrm{d} \omega \leqslant \frac{\psi\left(\mu_{1}\right)+\psi\left(\mu_{2}\right)}{2} .
$$

Definition 2.2. [16] A function $\psi: \mathrm{I} \subset(0,+\infty) \rightarrow \mathbb{R}$ is called $p$-convex, if

$$
\psi\left(M_{p}\left(\mu_{1}, \mu_{2} ; \omega\right)\right) \leqslant \omega \psi\left(\mu_{1}\right)+(1-\omega) \psi\left(\mu_{2}\right),
$$

$\forall \mu_{1}, \mu_{2} \in I$ and $\omega \in[0,1]$, where $p \in \mathbb{R} \backslash\{0\}$. If the above inequality is reverse, then $\psi$ is $p$-concave.

we add some comments on the above definition, if we choose $p=1$, then it collapses to classical convex functions, and in the case of $p=-1$, then the above definition collapses to harmonically convex functions.

As of late, Saima et al. [17] introduced a generalization form of convexity namely s-type convex function.

Definition 2.3. [17] A real-valued function $\psi: \mathbb{A} \rightarrow \mathbb{R}$ is said to be s-type convex function, if

$$
\psi\left(\omega \mu_{1}+(1-\omega) \mu_{2}\right) \leqslant[1-s(1-\omega)] \psi\left(\mu_{1}\right)+[1-s \omega] \psi\left(\mu_{2}\right),
$$

holds $\forall \mu_{1}, \mu_{2} \in \mathbb{A}, s \in[0,1]$ and $\omega \in[0,1]$.

In literature, the word "hypergeometric function" once in a while is summed up as a generalized hypergeometric function. Arnold Sommerfeld [18] was the first one who investigated the hypergeometric function by using differential equations in 1949. Kratzer and Franz investigated the hypergeometric function in terms of hypergeometric series. Lebedev in 1963, explored the hypergeometric function through the $\gamma$ function and its logarithmic derivative. In 2006, W. Van Assche [19] presented the Gauss hypergeometric function defined for $|z|<1$ by the hypergeometric series. It is regularly addressed by $\mathrm{F}(\mathrm{a}, \mathrm{b} ; \mathrm{c} ; z)$. The famous mathematician Euler was the one who for the first time explored the integral version of the hypergeometric function. Since then it has been the hot topic for researchers because of its huge applications in physics, number theory, finite vector spaces, cylinder and spherical problems, etc.

We remember the hypergeometric function [20], which is defined by Euler in integral form.

$$
{ }_{2} F_{1}(a, b ; c ; z)=\frac{1}{\beta(b, c-b)} \int_{0}^{1} \omega^{b-1}(1-\omega)^{c-b-1}(1-z \omega)^{-a} d \omega, c>b>0,|z|<1 .
$$

Owing to the aforementioned trend and inspired by the ongoing activities, we organize the paper in the following pattern, first of all, we give the idea about newly concept namely s-type $p$-convex functions. Secondly, we will attain some refinements of the Ostrowski type inequalities regarding the newly introduced idea. 


\section{Generalized $p$-convex functions and properties}

The main objective and aim of this section are to introduce and define a new definition called s-type $\mathrm{p}$-convex functions.

Definition 3.1. A nonnegative function $\psi: \mathbb{A}=\left[\mu_{1}, \mu_{2}\right] \rightarrow \mathbb{R}$ is called s-type $p$-convex, if

$$
\psi\left(M_{p}\left(\mu_{1}, \mu_{2} ; \omega\right)\right) \leqslant[1-(s(1-\omega))] \psi\left(\mu_{1}\right)+[1-(s \omega)] \psi\left(\mu_{2}\right),
$$

holds for every $\mu_{1}, \mu_{2} \in \mathbb{A}, s \in[0,1]$, and $\omega \in[0,1]$.

Remark 3.2. (i) Choosing $p=1$ in definition 3.1, then we get a definition namely s-type convex function which is defined by İscan et al. [17].

(ii) Choosing $p=-1$ in definition 3.1, we obtain the following new definition about harmonic s-type convex function:

$$
\psi\left(\frac{\mu_{1} \mu_{2}}{\omega \mu_{2}+(1-\omega) \mu_{1}}\right) \leqslant[1-(s(1-\omega))] \psi\left(\mu_{1}\right)+[1-(s \omega)] \psi\left(\mu_{2}\right) .
$$

(iii) Choosing $p=1$ and $s=1$ in definition 3.1, we get Definition 2.1.

(iv) Choosing $p=-1$ and $s=1$ in definition 3.1, then we attain a İşcan et al. [21] published definition namely harmonically convex function.

Lemma 3.3. The following inequalities $[1-(s(1-\omega))] \geqslant \omega$ and $[1-(s \omega)] \geqslant(1-\omega)$ are hold. If for all $\omega \in[0,1]$.

Proof. The proof is evident.

Proposition 3.4. Every $\mathrm{p}$-convex function on a $\mathrm{p}$-convex set is an $\mathrm{s}$-type $\mathrm{p}$-convex function.

Proof. Using the definition of $p$-convex function and from the lemma 3.3 , since $\omega \leqslant$ $[1-(s(1-\omega))]$ and $(1-\omega) \leqslant[1-(s \omega)]$ for all $\omega \in[0,1]$, we have

$$
\begin{aligned}
& \psi\left(M_{p}\left(\mu_{1}, \mu_{2} ; \omega\right)\right) \leqslant \omega \psi\left(\mu_{1}\right)+(1-\omega) \psi\left(\mu_{2}\right) \\
& \leqslant(1-(s(1-\omega))) \psi\left(\mu_{1}\right)+(1-(s \omega)) \psi\left(\mu_{2}\right) .
\end{aligned}
$$

Proposition 3.5. Every s-type $\mathrm{p}$-convex function is an $\mathrm{h}$-convex function with $\mathrm{h}(\omega)=[1-$ $(s(1-\omega))]$.

Proof.

$$
\begin{gathered}
\psi\left(M_{p}\left(\mu_{1}, \mu_{2} ; \omega\right)\right) \leqslant(1-(s(1-\omega))) \psi\left(\mu_{1}\right)+(1-(s \omega)) \psi\left(\mu_{2}\right) \\
\leqslant h(\omega) \psi\left(\mu_{1}\right)+h(1-\omega) \psi\left(\mu_{2}\right) .
\end{gathered}
$$

Now we makes some examples via newly introduce definition s-type $p$-convex function. 
Example 3.6. If $\psi(x)=x^{p}$ is $p$-convex function for all positive values of $x$ and $p \in$ $(-\infty, 0) \cup[1, \infty)[22]$, then by using Proposition 3.4, it is s-type $p$-convex function.

Example 3.7. Let $\psi:(0, \infty) \rightarrow \mathbb{R}, \psi(x)=x^{-p}, p \geqslant 1$, then $\psi$ is $p$-convex function [22], so by using Proposition 3.4, it is $s$-type $p$-convex function.

These are the clear advantages of the proposed new definition with respect to other known functions on the topic mentioned above.

Now, we will study some of its algebraic properties.

Theorem 3.8. Sum of two s-type p-convex functions is again s-type p-convex function.

Proof. Let $\psi$ and $\varphi$ be s-type $p$-convex, then

$$
\begin{aligned}
& (\psi+\varphi)\left(M_{p}\left(\mu_{1}, \mu_{2} ; \omega\right)\right) \\
& =\psi\left(M_{p}\left(\mu_{1}, \mu_{2} ; \omega\right)\right)+\varphi\left(M_{\mathfrak{p}}\left(\mu_{1}, \mu_{2} ; \omega\right)\right) \\
& \leqslant(1-(s(1-\omega))) \psi\left(\mu_{1}\right)+(1-(s \omega)) \psi\left(\mu_{2}\right) \\
& +(1-(s(1-\omega))) \varphi\left(\mu_{1}\right)+(1-(s \omega)) \varphi\left(\mu_{2}\right) \\
& =(1-(s(1-\omega)))\left[\psi\left(\mu_{1}\right)+\varphi\left(\mu_{1}\right)\right]+(1-(s \omega))\left[\psi\left(\mu_{2}\right)+\varphi\left(\mu_{2}\right)\right] \\
& =(1-(s(1-\omega)))(\psi+\varphi)\left(\mu_{1}\right)+(1-(s \omega))(\psi+\varphi)\left(\mu_{2}\right),
\end{aligned}
$$

which completes the proof.

Theorem 3.9. Scalar multiplication of s-type $\mathrm{p}$-convex functions is again s-type $\mathrm{p}$-convex function.

Proof. Let $\psi$ be s-type $p$-convex function and $c \in \mathbb{R}$ then

$$
\begin{aligned}
& (\mathrm{c} \psi)\left(M_{\mathfrak{p}}\left(\mu_{1}, \mu_{2} ; \omega\right)\right) \\
& \leqslant c\left[(1-(s(1-\omega))) \psi\left(\mu_{1}\right)+(1-(s \omega)) \psi\left(\mu_{2}\right)\right] \\
& =(1-(s(1-\omega))) c \psi\left(\mu_{1}\right)+(1-(s \omega)) c \psi\left(\mu_{2}\right) \\
& =(1-(s(1-\omega)))(c \psi)\left(\mu_{1}\right)+(1-(s \omega))(c \psi)\left(\mu_{2}\right),
\end{aligned}
$$

which completes the proof.

Theorem 3.10. Let $\varphi: \mathrm{I} \rightarrow \mathrm{J}$ be $\mathrm{p}$-convex function and $\psi: \mathrm{J} \rightarrow \mathbb{R}$ is non-decreasing and s-type convex function. Then the composition of these functions is $s$-type $p$-convex function.

Proof. For all $\mu_{1}, \mu_{2} \in \mathrm{I}$, and $\omega \in[0,1]$, we have

$$
\begin{aligned}
& (\psi \circ \varphi)\left(M_{p}\left(\mu_{1}, \mu_{2} ; \omega\right)\right) \\
& =\psi\left(\varphi\left(M_{p}\left(\mu_{1}, \mu_{2} ; \omega\right)\right)\right) \\
& \leqslant \psi\left(\omega \varphi\left(\mu_{1}\right)+(1-\omega) \varphi\left(\mu_{2}\right)\right) \\
& \leqslant(1-(s(1-\omega))) \psi\left(\varphi\left(\mu_{1}\right)\right)+(1-(s \omega)) \psi\left(\varphi\left(\mu_{2}\right)\right) \\
& =(1-(s(1-\omega)))(\psi \circ \varphi)\left(\mu_{1}\right)+(1-(s \omega))(\psi \circ \varphi)\left(\mu_{2}\right),
\end{aligned}
$$

which completes the proof. 


\section{Ostrowski type inequalities via Generalized $p$-convex function}

The main objective of this section is to attain and find some new Ostrowski type inequalities in the manner of the newly introduced definition. For attaining the results, we use the following lemma.

Lemma 4.1. [22] Let $\psi: \mathbb{A} \subseteq(0, \infty) \rightarrow \mathbb{R}$ be differential mapping on $\mathbb{A}^{\circ}$, where $\mu_{1}, \mu_{2} \in \mathbb{A}$ with $\mu_{1}<\mu_{2}$ and $p \in \mathbb{R} \backslash\{0\}$. If $\psi^{\prime} \in \mathrm{L}\left[\mu_{1}, \mu_{2}\right]$, then

$$
\begin{aligned}
& \psi(x)-\frac{p}{\mu_{2}^{p}-\mu_{1}^{p}} \int_{\mu_{1}}^{\mu_{2}} \frac{\psi(u)}{u^{1-p}} d u \\
& =\frac{1}{p\left(\mu_{2}^{p}-\mu_{1}^{p}\right)}\left\{\left(x^{p}-\mu_{1}^{p}\right)^{2} \int_{0}^{1} M_{p}^{-1}\left(x, \mu_{1} ; \omega\right)(\omega) \psi^{\prime}\left(M_{p}\left(x, \mu_{1} ; \omega\right)\right) d \omega\right. \\
& -\left\{\left(\mu_{2}^{p}-x^{p}\right)^{2} \int_{0}^{1} M_{p}^{-1}\left(x, \mu_{2} ; \omega\right)(\omega) \psi^{\prime}\left(M_{p}\left(x, \mu_{2} ; \omega\right)\right) d \omega\right\},
\end{aligned}
$$

for each $x \in\left[\mu_{1}, \mu_{2}\right]$ and where $M_{p}^{-1}\left(x, \mu_{1} ; \omega\right)=\left[\omega x^{p}+(1-\omega) \mu_{1}^{p}\right]^{\frac{1}{p}-1}$ and $M_{p}^{-1}\left(x, \mu_{2} ; \omega\right)=$ $\left[\omega x^{p}+(1-\omega) \mu_{2}\right]^{\frac{1}{p}-1}$.

Theorem 4.2. Let $\psi: \mathbb{A} \subseteq(0, \infty) \rightarrow \mathbb{R}$ be differential mapping on $\mathbb{A}^{\circ}$, where $\mu_{1}, \mu_{2} \in \mathbb{A}$ with $\mu_{1}<\mu_{2}, p \in \mathbb{R} \backslash\{0\}$ and $\psi^{\prime} \in \mathrm{L}\left[\mu_{1}, \mu_{2}\right]$. If $\left|\psi^{\prime}\right|^{\mathrm{q}}$ is s-type $\mathrm{p}$-convex for $\mathrm{p}>0, \mathrm{q} \geqslant 1$ and $s \in[0,1]$, then $\forall x \in\left[\mu_{1}, \mu_{2}\right]$, we have

$$
\begin{aligned}
& \left|\psi(x)-\frac{p}{\mu_{2}^{p}-\mu_{1}^{p}} \int_{\mu_{1}}^{\mu_{2}} \frac{\psi(u)}{u^{1-p}} d u\right| \\
& \leqslant \frac{\left(x^{p}-\mu_{1}^{p}\right)^{2}}{p\left(\mu_{2}^{p}-\mu_{1}^{p}\right)}\left(\frac{\beta(1,2)}{x^{p-1}}{ }_{2} F_{1}\left(1-\frac{1}{p^{2}}, 1 ; 3 ; 1-\frac{\mu_{1}^{p}}{x^{p}}\right)\right)^{1-\frac{1}{q}}\left(\Delta_{1}\left|\psi^{\prime}\left(\mu_{1}\right)\right|^{q}+\Delta_{2}\left|\psi^{\prime}\left(\mu_{2}\right)\right|^{q}\right)^{\frac{1}{q}} \\
& +\frac{\left(\mu_{2}^{p}-x^{p}\right)^{2}}{p\left(\mu_{2}^{p}-\mu_{1}^{p}\right)}\left(\frac{\beta(2,1)}{\mu_{2}^{p-1}}{ }_{2} F_{1}\left(1-\frac{1}{p^{2}}, 2 ; 3 ; 1-\frac{x^{p}}{\mu_{2}^{p}}\right)\right)^{1-\frac{1}{q}}\left(\Delta_{3}\left|\psi^{\prime}\left(\mu_{1}\right)\right|^{q}+\Delta_{4}\left|\psi^{\prime}\left(\mu_{2}\right)\right|^{q}\right)^{\frac{1}{q}},
\end{aligned}
$$

where

$$
\begin{aligned}
& \Delta_{1}=\frac{\beta(1,2)}{\chi^{p-1}}{ }_{2} F_{1}\left(1-\frac{1}{p}, 1 ; 3 ; 1-\frac{\mu_{1}^{p}}{\chi^{p}}\right)-s \frac{\beta(2,2)}{\chi^{p-1}}{ }_{2} F_{1}\left(1-\frac{1}{p^{p}}, 2 ; 4 ; 1-\frac{\mu_{1}^{p}}{\chi^{p}}\right), \\
& \Delta_{2}=\frac{\beta(1,2)}{\chi^{p-1}}{ }_{2} F_{1}\left(1-\frac{1}{p^{\prime}}, 1 ; 3 ; 1-\frac{\mu_{1}^{p}}{\chi^{p}}\right)-s \frac{\beta(1,3)}{x^{p-1}}{ }_{2} F_{1}\left(1-\frac{1}{p^{p}}, 1 ; 4 ; 1-\frac{\mu_{1}^{p}}{\chi^{p}}\right), \\
& \Delta_{3}=\frac{\beta(2,1)}{\mu_{2}^{p-1}}{ }_{2} F_{1}\left(1-\frac{1}{p^{2}}, 2 ; 3 ; 1-\frac{\chi^{p}}{\mu_{2}^{p}}\right)-s \frac{\beta(2,2)}{\mu_{2}^{p-1}}{ }_{2} F_{1}\left(1-\frac{1}{p^{\prime}}, 2 ; 4 ; 1-\frac{x^{p}}{\mu_{2}^{p}}\right) \text {, } \\
& \Delta_{4}=\frac{\beta(2,1)}{\mu_{2}^{p-1}}{ }_{2} F_{1}\left(1-\frac{1}{p^{2}}, 2 ; 3 ; 1-\frac{\mu_{2}^{p}}{\chi^{p}}\right)-s \frac{\beta(3,1)}{\mu_{2}^{p-1}}{ }_{2} F_{1}\left(1-\frac{1}{p^{\prime}}, 3 ; 4 ; 1-\frac{x^{p}}{\mu_{2}^{p}}\right) \text {. }
\end{aligned}
$$


Proof. Applying Lemma 4.1, properties of modulus, power mean inequality and the property of $s$-type $p$-convexity of $\left|\psi^{\prime}\right|^{q}$, we have

$$
\begin{aligned}
& \left|\psi(x)-\frac{p}{\mu_{2}^{p}-\mu_{1}^{p}} \int_{\mu_{1}}^{\mu_{2}} \frac{\psi(u)}{u^{1-p}} d u\right| \\
& =\frac{1}{p\left(\mu_{2}^{p}-\mu_{1}^{p}\right)}\left\{\left(x^{p}-\mu_{1}^{p}\right)^{2} \int_{0}^{1} M_{p}^{-1}\left(x, \mu_{1} ; \omega\right)(\omega)\left|\psi^{\prime}\left(M_{p}\left(x, \mu_{1} ; \omega\right)\right)\right| d \omega\right. \\
& +\left\{\left(\mu_{2}^{p}-x^{p}\right)^{2} \int_{0}^{1} M_{p}^{-1}\left(x, \mu_{2} ; \omega\right)(\omega)\left|\psi^{\prime}\left(M_{p}\left(x, \mu_{2} ; \omega\right)\right)\right| d \omega\right\} \\
& \leqslant \frac{\left(x^{p}-\mu_{1}^{p}\right)^{2}}{p\left(\mu_{2}^{p}-\mu_{1}^{p}\right)} \\
& \times\left(\int_{0}^{1} M_{p}^{-1}\left(x, \mu_{1} ; \omega\right)(\omega) d \omega\right)^{1-\frac{1}{q}}\left(\int_{0}^{1} M_{p}^{-1}\left(x, \mu_{1} ; \omega\right)(\omega)\left|\psi^{\prime}\left(M_{p}\left(x, \mu_{1} ; \omega\right)\right)\right|^{q} d \omega\right)^{\frac{1}{q}} \\
& +\frac{\left(\mu_{2}^{p}-\chi^{p}\right)^{2}}{p\left(\mu_{2}^{p}-\mu_{1}^{p}\right)} \\
& \times\left(\int_{0}^{1} M_{p}^{-1}\left(x, \mu_{2} ; \omega\right)(\omega) d \omega\right)^{1-\frac{1}{q}}\left(\int_{0}^{1} M_{p}^{-1}\left(x, \mu_{2} ; \omega\right)(\omega)\left|\psi^{\prime}\left(M_{p}\left(x, \mu_{2} ; \omega\right)\right)\right|^{q} d \omega\right)^{\frac{1}{q}} \\
& \leqslant \frac{\left(x^{p}-\mu_{1}^{p}\right)^{2}}{p\left(\mu_{2}^{p}-\mu_{1}^{p}\right)}\left(\int_{0}^{1} M_{p}^{-1}\left(x, \mu_{1} ; \omega\right)(\omega) d \omega\right)^{1-\frac{1}{q}} \\
& \times\left(\int_{0}^{1} M_{p}^{-1}\left(x, \mu_{1} ; \omega\right)(\omega)\left[[1-(s(1-\omega))]\left|\psi^{\prime}(x)\right|^{q}+[1-s \omega]\left|\psi^{\prime}\left(\mu_{1}\right)\right|^{q}\right] d \omega\right)^{\frac{1}{q}} \\
& +\frac{\left(\mu_{2}^{p}-x^{p}\right)^{2}}{p\left(\mu_{2}^{p}-\mu_{1}^{p}\right)}\left(\int_{0}^{1} M_{p}^{-1}\left(x, \mu_{2} ; \omega\right)(\omega) d \omega\right)^{1-\frac{1}{q}} \\
& \times\left(\int_{0}^{1} M_{p}^{-1}\left(x, \mu_{2} ; \omega\right)(\omega)\left[[1-(s(1-\omega))]\left|\psi^{\prime}(x)\right|^{q}+[1-s \omega]\left|\psi^{\prime}\left(\mu_{2}\right)\right|^{q}\right] d \omega\right)^{\frac{1}{q}} \\
& \leqslant \frac{\left(x^{p}-\mu_{1}^{p}\right)^{2}}{p\left(\mu_{2}^{p}-\mu_{1}^{p}\right)}\left(\int_{0}^{1} M_{p}^{-1}\left(x, \mu_{1} ; \omega\right)(\omega) d \omega\right)^{1-\frac{1}{q}}\left(\left|\psi^{\prime}(x)\right|^{q} \int_{0}^{1} M_{p}^{-1}\left(x, \mu_{1} ; \omega\right)(\omega)[1-(s(1-\omega))] d \omega\right. \\
& \left.+\left|\psi^{\prime}\left(\mu_{1}\right)\right|^{q} \int_{0}^{1} M_{p}^{-1}\left(x, \mu_{1} ; \omega\right)(\omega)[1-s \omega] d \omega\right)^{\frac{1}{q}} \\
& +\frac{\left(\mu_{2}^{p}-x^{p}\right)^{2}}{p\left(\mu_{2}^{p}-\mu_{1}^{p}\right)}\left(\int_{0}^{1} M_{p}^{-1}\left(x, \mu_{2} ; \omega\right)(\omega) d \omega\right)^{1-\frac{1}{q}}\left(\left|\psi^{\prime}(x)\right|^{q} \int_{0}^{1} M_{p}^{-1}\left(x, \mu_{2} ; \omega\right)(\omega)[1-(s(1-\omega))] d \omega\right. \\
& \left.+\left|\psi^{\prime}\left(\mu_{2}\right)\right|^{q} \int_{0}^{1} M_{p}^{-1}\left(x, \mu_{2} ; \omega\right)(\omega)[1-s \omega] d \omega\right)^{\frac{1}{q}} \\
& \leqslant \frac{\left(x^{p}-\mu_{1}^{p}\right)^{2}}{p\left(\mu_{2}^{p}-\mu_{1}^{p}\right)}\left(\frac{\beta(1,2)}{x^{p-1}}{ }_{2} F_{1}\left(1-\frac{1 s}{p}, 1 ; 3 ; 1-\frac{a^{p}}{x^{p}}\right)\right)^{1-\frac{1}{q}}\left(\Delta_{1}\left|\psi^{\prime}(x)\right|^{q}+\Delta_{2}\left|\psi^{\prime}\left(\mu_{1}\right)\right|^{q}\right)^{\frac{1}{q}} \\
& +\frac{\left(\mu_{2}^{p}-x^{p}\right)^{2}}{p\left(\mu_{2}^{p}-\mu_{1}^{p}\right)}\left(\frac{\beta(2,1)}{\mu_{2}^{p}-1}{ }_{2} F_{1}\left(1-\frac{1}{p^{\prime}}, 2 ; 3 ; 1-\frac{x^{p}}{\mu_{2}^{p}}\right)\right)^{1-\frac{1}{q}}\left(\Delta_{3}\left|\psi^{\prime}(x)\right|^{q}+\Delta_{4}\left|\psi^{\prime}\left(\mu_{2}\right)\right|^{q}\right)^{\frac{1}{q}} \text {, }
\end{aligned}
$$


which completes the proof.

Theorem 4.3. Let $\psi: \mathbb{A} \subseteq(0, \infty) \rightarrow \mathbb{R}$ be differential mapping on $\mathbb{A}^{\circ}$, where $\mu_{1}, \mu_{2} \in \mathbb{A}$ with $\mu_{1}<\mu_{2}, p \in \mathbb{R} \backslash\{0\}$ and $\psi^{\prime} \in \mathrm{L}\left[\mu_{1}, \mu_{2}\right]$. If $\left|\psi^{\prime}\right|^{q}$ is s-type $p$-convex for $s \in[0,1]$ and $q \geqslant 1$, then $\forall x \in\left[\mu_{1}, \mu_{2}\right]$, we have

$$
\begin{aligned}
& \left|\psi(x)-\frac{p}{\mu_{2}^{p}-\mu_{1}^{p}} \int_{\mu_{1}}^{\mu_{2}} \frac{\psi(u)}{u^{1-p}} d u\right| \\
& \leqslant \frac{\left(\mu_{2}^{p}-x^{p}\right)^{2}}{p\left(\mu_{2}^{p}-\mu_{1}^{p}\right)}\left(\frac{\beta(2,1)}{\mu_{1}^{p-1}}{ }_{2} F_{1}\left(1-\frac{1}{p^{2}}, 2 ; 3 ; 1-\frac{x^{p}}{\mu_{1}^{p}}\right)\right)^{1-\frac{1}{q}}\left(\Delta_{1}^{\prime}\left|\psi^{\prime}(x)\right|^{q}+\Delta_{2}^{\prime}\left|\psi^{\prime}\left(\mu_{1}\right)\right|^{q}\right)^{\frac{1}{q}} \\
& +\frac{\left(\mu_{2}^{p}-x^{p}\right)^{2}}{p\left(\mu_{2}^{p}-\mu_{1}^{p}\right)}\left(\frac{\beta(1,2)}{x^{p-1}}{ }_{2} F_{1}\left(1-\frac{1}{p^{\prime}}, 1 ; 3 ; 1-\frac{\mu_{2}^{p}}{x^{p}}\right)\right)^{1-\frac{1}{q}}\left(\Delta_{3}^{\prime}\left|\psi^{\prime}(x)\right|^{q}+\Delta_{4}^{\prime}\left|\psi^{\prime}\left(\mu_{2}\right)\right|^{q}\right)^{\frac{1}{q}},
\end{aligned}
$$

where

$$
\begin{aligned}
& \Delta_{1}^{\prime}=\frac{\beta(2,1)}{\mu_{1}^{p-1}}{ }_{2} \mathrm{~F}_{1}\left(1-\frac{1}{p^{2}}, 2 ; 3 ; 1-\frac{\chi^{p}}{\mu_{1}^{p}}\right)-s \frac{\beta(2,2)}{\mu_{1}^{p-1}}{ }_{2} \mathrm{~F}_{1}\left(1-\frac{1}{p^{2}}, 2 ; 3 ; 1-\frac{\chi^{p}}{\mu_{1}^{p}}\right), \\
& \Delta_{2}^{\prime}=\frac{\beta(2,1)}{\mu_{1}^{p-1}}{ }_{2} \mathrm{~F}_{1}\left(1-\frac{1}{p^{\prime}}, 2 ; 3 ; 1-\frac{\chi^{p}}{\mu_{1}^{p}}\right)-s \frac{\beta(3,1)}{\mu_{1}^{p-1}}{ }_{2} \mathrm{~F}_{1}\left(1-\frac{1}{p^{2}}, 3 ; 4 ; 1-\frac{\chi^{p}}{\mu_{1}^{p}}\right), \\
& \Delta_{3}^{\prime}=\frac{\beta(1,2)}{\chi^{p-1}}{ }_{2} F_{1}\left(1-\frac{1}{p^{p}}, 1 ; 3 ; 1-\frac{\mu_{2}^{p}}{\chi^{p}}\right)-s \frac{\beta(2,2)}{\chi^{p-1}}{ }_{2} F_{1}\left(1-\frac{1}{p^{p}}, 2 ; 4 ; 1-\frac{\mu_{2}^{p}}{\chi^{p}}\right), \\
& \Delta_{4}^{\prime}=\frac{\beta(1,2)}{\chi^{p-1}}{ }_{2} F_{1}\left(1-\frac{1}{p^{\prime}}, 1 ; 3 ; 1-\frac{\mu_{2}^{p}}{\chi^{p}}\right)-s \frac{\beta(1,3)}{\chi^{p-1}}{ }_{2} F_{1}\left(1-\frac{1}{p^{p}}, 1 ; 4 ; 1-\frac{\mu_{2}^{p}}{\chi^{p}}\right) .
\end{aligned}
$$

Proof. The proof is omitted as it is similar to the proof of Theorem 4.2. If we are choosing $p<0$, then as a result we get the inequality (4.1) is easily attained.

Theorem 4.4. Let $\psi: \mathbb{A} \subseteq(0, \infty) \rightarrow \mathbb{R}$ be differential mapping on $\mathbb{A}^{\circ}$, where $\mu_{1}, \mu_{2} \in \mathbb{A}$ with $\mu_{1}<\mu_{2}, p \in \mathbb{R} \backslash\{0\}$ and $\psi^{\prime} \in \mathrm{L}\left[\mu_{1}, \mu_{2}\right]$. If $\left|\psi^{\prime}\right|^{\mathrm{q}}$ is s-type $\mathrm{p}$-convex for $\mathrm{p}>0, \mathrm{~s} \in[0,1]$ and $\mathrm{q} \geqslant 1$, then $\forall x \in\left[\mu_{1}, \mu_{2}\right]$, we have

$$
\begin{aligned}
& \left|\psi(x)-\frac{p}{\mu_{2}^{p}-\mu_{1}^{p}} \int_{\mu_{1}}^{\mu_{2}} \frac{\psi(u)}{u^{1-p}} d u\right| \\
& \leqslant \frac{\left(x^{p}-\mu_{1}^{p}\right)^{2}}{p\left(\mu_{2}^{p}-\mu_{1}^{p}\right)}\left(\frac{\beta(1,1)}{x^{p-1}}{ }_{2} F_{1}\left(1-\frac{1}{p^{\prime}}, 1 ; 2 ; 1-\frac{\mu_{1}^{p}}{x^{p}}\right)\right)^{1-\frac{1}{q}}\left(\Delta_{5}\left|\psi^{\prime}(x)\right|^{q}+\Delta_{6}\left|\psi^{\prime}\left(\mu_{1}\right)\right|^{q}\right)^{\frac{1}{q}} \\
& +\frac{\left(\mu_{2}^{p}-x^{p}\right)^{2}}{p\left(\mu_{2}^{p}-\mu_{1}^{p}\right)}\left(\frac{\beta(1,1)}{\mu_{2}^{p-1}}{ }_{2} F_{1}\left(1-\frac{1}{p^{2}}, 1 ; 2 ; 1-\frac{x^{p}}{\mu_{2}^{p}}\right)\right)^{1-\frac{1}{q}}\left(\Delta_{7}\left|\psi^{\prime}(x)\right|^{q}+\Delta_{8}\left|\psi^{\prime}\left(\mu_{2}\right)\right|^{q}\right)^{\frac{1}{q}}
\end{aligned}
$$

where

$$
\begin{aligned}
& \Delta_{5}=\frac{\beta(1, q+1)}{x^{p-1}}{ }_{2} F_{1}\left(1-\frac{1}{p^{\prime}}, 1 ; q+2 ; 1-\frac{\mu_{1}^{p}}{x^{p}}\right)-s \frac{\beta(2, q+1)}{x^{p-1}}{ }_{2} F_{1}\left(1-\frac{1}{p^{2}}, 2 ; q+3 ; 1-\frac{\mu_{1}^{p}}{\chi^{p}}\right), \\
& \Delta_{6}=\frac{\beta(1, q+1)}{x^{p-1}}{ }_{2} F_{1}\left(1-\frac{1}{p^{p}}, 1 ; q+2 ; 1-\frac{\mu_{1}^{p}}{x^{p}}\right)-s \frac{\beta(1, q+2)}{x^{p-1}}{ }_{2} F_{1}\left(1-\frac{1}{p^{p}}, 1 ; q+3 ; 1-\frac{\mu_{1}^{p}}{x^{p}}\right),
\end{aligned}
$$




$$
\begin{aligned}
& \Delta_{7}=\frac{\beta(q+1,1)}{\mu_{2}^{p-1}}{ }_{2} F_{1}\left(1-\frac{1}{p^{\prime}}, q+1 ; q+2 ; 1-\frac{x^{p}}{\mu_{2}^{p}}\right) \\
& -s \frac{\beta(q+1,2)}{\mu_{2}^{p-1}}{ }_{2} F_{1}\left(1-\frac{1}{p^{\prime}}, q+1 ; q+3 ; 1-\frac{x^{p}}{\mu_{2}^{p}}\right) \text {, } \\
& \Delta_{8}=\frac{\beta(\mathrm{q}+1,1)}{\mu_{2}^{p-1}}{ }_{2} \mathrm{~F}_{1}\left(1-\frac{1}{\mathrm{p}^{\prime}}, \mathrm{q}+1 ; \mathrm{q}+2 ; 1-\frac{\mu_{2}^{\mathrm{p}}}{\chi^{\mathrm{p}}}\right) \\
& -s \frac{\beta(q+2,1)}{\mu_{2}^{p-1}}{ }_{2} F_{1}\left(1-\frac{1}{p^{2}}, q+2 ; q+3 ; 1-\frac{x^{p}}{\mu_{2}^{p}}\right) \text {. }
\end{aligned}
$$

Proof. Applying Lemma 4.1, properties of modulus, power mean integral inequality and property of $s$-type $p$-convexity of $\left|\psi^{\prime}\right|^{q}$, we have

$$
\begin{aligned}
& \left|\psi(x)-\frac{p}{\mu_{2}^{p}-\mu_{1}^{p}} \int_{\mu_{1}}^{\mu_{2}} \frac{\psi(u)}{u^{1-p}} d u\right| \\
& =\frac{1}{p\left(\mu_{2}^{p}-\mu_{1}^{p}\right)}\left\{\left(x^{p}-\mu_{1}^{p}\right)^{2} \int_{0}^{1} M_{p}^{-1}\left(x, \mu_{1} ; \omega\right)(\omega)\left|\psi^{\prime}\left(M_{p}\left(x, \mu_{1} ; \omega\right)\right)\right| d \omega\right. \\
& +\left\{\left(\mu_{2}^{p}-x^{p}\right)^{2} \int_{0}^{1} M_{p}^{-1}\left(x, \mu_{2} ; \omega\right)(\omega)\left|\psi^{\prime}\left(M_{p}\left(x, \mu_{2} ; \omega\right)\right)\right| d \omega\right\} \\
& \leqslant \frac{\left(x^{p}-\mu_{1}^{p}\right)^{2}}{p\left(\mu_{2}^{p}-\mu_{1}^{p}\right)} \\
& \times\left(\int_{0}^{1} M_{p}^{-1}\left(x, \mu_{1} ; \omega\right) d \omega\right)^{1-\frac{1}{q}}\left(\int_{0}^{1} M_{p}^{-1}\left(x, \mu_{1} ; \omega\right) \omega^{q}\left|\psi^{\prime}\left(M_{p}\left(x, \mu_{1} ; \omega\right)\right)\right|^{q} d \omega\right)^{\frac{1}{q}} \\
& +\frac{\left(\mu_{2}^{p}-x^{p}\right)^{2}}{p\left(\mu_{2}^{p}-\mu_{1}^{p}\right)} \\
& \times\left(\int_{0}^{1} M_{p}^{-1}\left(x, \mu_{2} ; \omega\right) d \omega\right)^{1-\frac{1}{q}}\left(\int_{0}^{1} M_{p}^{-1}\left(x, \mu_{2} ; \omega\right) \omega^{q}\left|\psi^{\prime}\left(M_{p}\left(x, \mu_{2} ; \omega\right)\right)\right|^{q} d \omega\right)^{\frac{1}{q}} \\
& \leqslant \frac{\left(x^{p}-\mu_{1}^{p}\right)^{2}}{p\left(\mu_{2}^{p}-\mu_{1}^{p}\right)}\left(\int_{0}^{1} M_{p}^{-1}\left(x, \mu_{1} ; \omega\right) d \omega\right)^{1-\frac{1}{q}} \\
& \times\left(\int_{0}^{1} M_{p}^{-1}\left(x, \mu_{1} ; \omega\right) \omega^{q}\left[[1-(s(1-\omega))]\left|\psi^{\prime}(x)\right|^{q}+[1-s \omega]\left|\psi^{\prime}\left(\mu_{1}\right)\right|^{q}\right] d \omega\right)^{\frac{1}{q}} \\
& +\frac{\left(\mu_{2}^{p}-x^{p}\right)^{2}}{p\left(\mu_{2}^{p}-\mu_{1}^{p}\right)}\left(\int_{0}^{1} M_{p}^{-1}\left(x, \mu_{2} ; \omega\right) d \omega\right)^{1-\frac{1}{q}} \\
& \times\left(\int_{0}^{1} M_{p}^{-1}\left(x, \mu_{2} ; \omega\right) \omega^{q}\left[[1-(s(1-\omega))]\left|\psi^{\prime}(x)\right|^{q}+[1-s \omega]\left|\psi^{\prime}\left(\mu_{2}\right)\right|^{q}\right] d \omega\right)^{\frac{1}{q}}
\end{aligned}
$$




$$
\begin{aligned}
& \leqslant \frac{\left(\chi^{p}-\mu_{1}^{p}\right)^{2}}{p\left(\mu_{2}^{p}-\mu_{1}^{p}\right)}\left(\int_{0}^{1} M_{p}^{-1}\left(x, \mu_{1} ; \omega\right) d \omega\right)^{1-\frac{1}{q}} \\
& \times\left(\left|\psi^{\prime}(x)\right|^{\mathrm{q}} \int_{0}^{1} M_{p}^{-1}\left(x, \mu_{1} ; \omega\right) \omega^{\mathrm{q}}[1-(s(1-\omega))] \mathrm{d} \omega\right. \\
& \left.+\left|\psi^{\prime}\left(\mu_{1}\right)\right|^{q} \int_{0}^{1} M_{p}^{-1}\left(x, \mu_{1} ; \omega\right) \omega^{q}[1-s \omega] d \omega\right)^{\frac{1}{q}} \\
& +\frac{\left(\mu_{2}^{p}-x^{p}\right)^{2}}{p\left(\mu_{2}^{p}-\mu_{1}^{p}\right)}\left(\int_{0}^{1} M_{p}^{-1}\left(x, \mu_{2} ; \omega\right) d \omega\right)^{1-\frac{1}{q}} \\
& \times\left(\left|\psi^{\prime}(x)\right|^{\mathrm{q}} \int_{0}^{1} M_{\mathrm{p}}^{-1}\left(x, \mu_{2} ; \omega\right) \omega^{\mathrm{q}}[1-(s(1-\omega))] \mathrm{d} \omega\right. \\
& \left.+\left|\psi^{\prime}\left(\mu_{2}\right)\right|^{q} \int_{0}^{1} M_{p}^{-1}\left(x, \mu_{2} ; \omega\right) \omega^{q}[1-s \omega] d \omega\right)^{\frac{1}{q}} \\
& \leqslant \frac{\left(x^{p}-\mu_{1}^{p}\right)^{2}}{p\left(\mu_{2}^{p}-\mu_{1}^{p}\right)}\left(\frac{\beta(1,1)}{x^{p-1}}{ }_{2} F_{1}\left(1-\frac{1}{p^{\prime}}, 1 ; 2 ; 1-\frac{\mu_{1}^{p}}{x^{p}}\right)\right)^{1-\frac{1}{q}}\left(\Delta_{5}\left|\psi^{\prime}(x)\right|^{q}+\Delta_{6}\left|\psi^{\prime}\left(\mu_{1}\right)\right|^{q}\right)^{\frac{1}{q}} \\
& +\frac{\left(\mu_{2}^{p}-x^{p}\right)^{2}}{p\left(\mu_{2}^{p}-\mu_{1}^{p}\right)}\left(\frac{\beta(1,1)}{\mu_{2}^{p-1}}{ }_{2} F_{1}\left(1-\frac{1}{p}, 1 ; 2 ; 1-\frac{x^{p}}{\mu_{2}^{p}}\right)\right)^{1-\frac{1}{q}}\left(\Delta_{7}\left|\psi^{\prime}(x)\right|^{q}+\Delta_{8}\left|\psi^{\prime}\left(\mu_{2}\right)\right|^{q}\right)^{\frac{1}{q}} \text {, }
\end{aligned}
$$

which completes the proof.

Theorem 4.5. Let $\psi: \mathbb{A} \subseteq(0, \infty) \rightarrow \mathbb{R}$ be differential mapping on $\mathbb{A}^{\circ}$, where $\mu_{1}, \mu_{2} \in \mathbb{A}$ with $\mu_{1}<\mu_{2}, p \in \mathbb{R} \backslash\{0\}$ and $\psi^{\prime} \in \mathrm{L}\left[\mu_{1}, \mu_{2}\right]$. If $\left|\psi^{\prime}\right|^{q}$ is s-type $p$-convex for $s \in[0,1]$ and $q \geqslant 1$, then $\forall x \in\left[\mu_{1}, \mu_{2}\right]$, we have

$$
\begin{aligned}
& \left|\psi(x)-\frac{p}{\mu_{2}^{p}-\mu_{1}^{p}} \int_{\mu_{1}}^{\mu_{2}} \frac{\psi(u)}{u^{1-p}} d u\right| \\
& \leqslant \frac{\left(x^{p}-\mu_{2}^{p}\right)^{2}}{p\left(\mu_{2}^{p}-\mu_{1}^{p}\right)}\left(\frac{\beta(1,1)}{x^{p-1}}{ }_{2} F_{1}\left(1-\frac{1}{p^{2}}, 1 ; 2 ; 1-\frac{\mu_{1}^{p}}{x^{p}}\right)\right)^{1-\frac{1}{q}}\left(\Delta_{5}^{\prime}\left|\psi^{\prime}(x)\right|^{q}+\Delta_{6}^{\prime}\left|\psi^{\prime}\left(\mu_{1}\right)\right|^{q}\right)^{\frac{1}{q}} \\
& +\frac{\left(\mu_{2}^{p}-x^{p}\right)^{2}}{p\left(\mu_{2}^{p}-\mu_{1}^{p}\right)}\left(\frac{\beta(1,1)}{\mu_{2}^{p-1}}{ }_{2} F_{1}\left(1-\frac{1}{p^{2}}, 1 ; 2 ; 1-\frac{x^{p}}{\mu_{2}^{p}}\right)\right)^{1-\frac{1}{q}}\left(\Delta_{7}^{\prime}\left|\psi^{\prime}(x)\right|^{q}+\Delta_{8}^{\prime}\left|\psi^{\prime}\left(\mu_{2}\right)\right|^{q}\right)^{\frac{1}{q}},
\end{aligned}
$$

where

$$
\begin{aligned}
& \Delta_{5}^{\prime}=\frac{\beta(\mathrm{q}+1,1)}{\mu_{1}^{p-1}}{ }_{2} \mathrm{~F}_{1}\left(1-\frac{1}{\mathrm{p}}, \mathrm{q}+1 ; \mathrm{q}+2 ; 1-\frac{\chi^{\mathrm{p}}}{\mu_{1}^{\mathrm{p}}}\right) \\
& -s \frac{\beta(q+1,2)}{\mu_{1}^{p-1}}{ }_{2} F_{1}\left(1-\frac{1}{p^{\prime}}, q+1 ; q+3 ; 1-\frac{x^{p}}{\mu_{1}^{p}}\right), \\
& \Delta_{6}^{\prime}=\frac{\beta(\mathrm{q}+1,1)}{\mu_{1}^{p-1}}{ }_{2} \mathrm{~F}_{1}\left(1-\frac{1}{\mathrm{p}^{\prime}}, \mathrm{q}+1 ; \mathrm{q}+2 ; 1-\frac{\chi^{\mathrm{p}}}{\mu_{1}^{\mathrm{p}}}\right) \\
& -s \frac{\beta(q+2,1)}{\mu_{1}^{p-1}}{ }_{2} F_{1}\left(1-\frac{1}{p^{\prime}}, q+2 ; q+3 ; 1-\frac{x^{p}}{\mu_{1}^{p}}\right) \text {, }
\end{aligned}
$$




$$
\begin{aligned}
& \Delta_{7}^{\prime}=\frac{\beta(1, q+1)}{x^{p-1}}{ }_{2} F_{1}\left(1-\frac{1}{p^{\prime}}, 1 ; q+2 ; 1-\frac{\mu_{2}^{p}}{\chi^{p}}\right)-s \frac{\beta(2, q+1)}{x^{p-1}}{ }_{2} F_{1}\left(1-\frac{1}{p^{\prime}}, 2 ; q+3 ; 1-\frac{\mu_{2}^{p}}{\chi^{p}}\right) \text {, } \\
& \Delta_{8}^{\prime}=\frac{\beta(1, q+1)}{x^{p-1}}{ }_{2} F_{1}\left(1-\frac{1}{p^{\prime}}, 1 ; q+2 ; 1-\frac{\mu_{2}^{p}}{x^{p}}\right)-s \frac{\beta(1, q+2)}{x^{p-1}}{ }_{2} F_{1}\left(1-\frac{1}{p^{\prime}}, 1 ; q+3 ; 1-\frac{\mu_{2}^{p}}{\chi^{p}}\right) \text {. }
\end{aligned}
$$

Proof. The proof is omitted as it is similar to the proof of Theorem 4.4. If we are choosing $p<0$, then as a result we get the inequality (4.2) is easily attained.

Theorem 4.6. Let $\psi: \mathbb{A} \subseteq(0, \infty) \rightarrow \mathbb{R}$ be differential mapping on $\mathbb{A}^{\circ}$, where $\mu_{1}, \mu_{2} \in \mathbb{A}$ with $\mu_{1}<\mu_{2}, p \in \mathbb{R} \backslash\{0\}$ and $\psi^{\prime} \in \mathrm{L}\left[\mu_{1}, \mu_{2}\right]$. If $\left|\psi^{\prime}\right|^{\mathrm{q}}$ is s-type $p$-convex for $s \in[0,1]$, $\mathrm{p}>0, \mathrm{q} \geqslant 1$, then $\forall x \in\left[\mu_{1}, \mu_{2}\right]$, we have

$$
\begin{aligned}
& \left|\psi(x)-\frac{p}{\mu_{2}^{p}-\mu_{1}^{p}} \int_{\mu_{1}}^{\mu_{2}} \frac{\psi(u)}{u^{1-p}} d u\right| \\
& \leqslant \frac{1}{p\left(\mu_{2}^{p}-\mu_{1}^{p}\right)} \times\left\{\left(x^{p}-\mu_{1}^{p}\right)^{2}\left(\frac{\beta(1,1)}{x^{r p-r}}{ }_{2} F_{1}\left(r-\frac{r}{p^{\prime}}, 1 ; 2 ; 1-\frac{\mu_{1}^{p}}{x^{p}}\right)\right)^{\frac{1}{r}}\right. \\
& \left(\left(\frac{1}{q+1}+s \beta(q+1,2)\right)\left|\psi^{\prime}(x)\right|^{q}+\left(\frac{1-s}{q+2}\right)\left|\psi^{\prime}\left(\mu_{1}\right)\right|^{q}\right)^{\frac{1}{q}} \\
& +\left(\mu_{2}^{p}-x^{p}\right)^{2}\left(\frac{\beta(1,1)}{\mu_{2}^{r p-r}}{ }_{2} F_{1}\left(r-\frac{r}{p^{\prime}} 1 ; 2 ; 1-\frac{x^{p}}{\mu_{2}^{p}}\right)\right)^{\frac{1}{r}} \\
& \left.\left(\left(\frac{1}{q+1}+s \beta(q+1,2)\right)\left|\psi^{\prime}(x)\right|^{q}+\left(\frac{1-s}{q+2}\right)\left|\psi^{\prime}\left(\mu_{2}\right)\right|^{q}\right)^{\frac{1}{q}}\right\} .
\end{aligned}
$$

Proof. Applying Lemma 4.1, properties of modulus, Höder's inequality and the property of $s$-type $p$-convexity of $\left|\psi^{\prime}\right|^{q}$, we have

$$
\begin{aligned}
& \left|\psi(x)-\frac{p}{\mu_{2}^{p}-\mu_{1}^{p}} \int_{\mu_{1}}^{\mu_{2}} \frac{\psi(u)}{u^{1-p}} d u\right| \\
& =\frac{1}{p\left(\mu_{2}^{p}-\mu_{1}^{p}\right)}\left\{\left(x^{p}-\mu_{1}^{p}\right)^{2} \int_{0}^{1} M_{p}^{-1}\left(x, \mu_{1} ; \omega\right)(\omega)\left|\psi^{\prime}\left(M_{p}\left(x, \mu_{1} ; \omega\right)\right)\right| d \omega\right. \\
& +\left\{\left(\mu_{2}^{p}-x^{p}\right)^{2} \int_{0}^{1} M_{p}^{-1}\left(x, \mu_{2} ; \omega\right)(\omega)\left|\psi^{\prime}\left(M_{p}\left(x, \mu_{2} ; \omega\right)\right)\right| d \omega\right\} \\
& \leqslant \frac{\left(x^{p}-\mu_{1}^{p}\right)^{2}}{p\left(\mu_{2}^{p}-\mu_{1}^{p}\right)}\left(\int_{0}^{1} M_{p}^{-r}\left(x, \mu_{1} ; \omega\right) d \omega\right)^{\frac{1}{r}}\left(\int_{0}^{1} \omega^{q}\left|\psi^{\prime}\left(M_{p}\left(x, \mu_{1} ; \omega\right)\right)\right|^{q} d \omega\right)^{\frac{1}{q}} \\
& +\frac{\left(\mu_{2}^{p}-x^{p}\right)^{2}}{p\left(\mu_{2}^{p}-\mu_{1}^{p}\right)}\left(\int_{0}^{1} M_{p}^{-r}\left(x, \mu_{2} ; \omega\right) d \omega\right)^{\frac{1}{r}}\left(\int_{0}^{1} \omega^{q}\left|\psi^{\prime}\left(M_{p}\left(x, \mu_{2} ; \omega\right)\right)\right|^{q} d \omega\right)^{\frac{1}{q}}
\end{aligned}
$$




$$
\begin{aligned}
& \leqslant \frac{\left(x^{p}-\mu_{1}^{p}\right)^{2}}{p\left(\mu_{2}^{p}-\mu_{1}^{p}\right)}\left(\int_{0}^{1} M_{p}^{-r}\left(x, \mu_{1} ; \omega\right) d \omega\right)^{\frac{1}{r}} \\
& \times\left(\int_{0}^{1} \omega^{\mathrm{q}}\left[[1-(s(1-\omega))]\left|\psi^{\prime}(x)\right|^{\mathrm{q}}+[1-s \omega]\left|\psi^{\prime}\left(\mu_{1}\right)\right|^{\mathrm{q}}\right] \mathrm{d} \omega\right)^{\frac{1}{q}} \\
& +\frac{\left(\mu_{2}^{p}-x^{p}\right)^{2}}{p\left(\mu_{2}^{p}-\mu_{1}^{p}\right)}\left(\int_{0}^{1} M_{p}^{-r}\left(x, \mu_{2} ; \omega\right) d \omega\right)^{\frac{1}{r}} \\
& \times\left(\int_{0}^{1} \omega^{\mathrm{q}}\left[[1-(s(1-\omega))]\left|\psi^{\prime}(x)\right|^{\mathrm{q}}+[1-s \omega]\left|\psi^{\prime}\left(\mu_{2}\right)\right|^{\mathrm{q}}\right] \mathrm{d} \omega\right)^{\frac{1}{q}} \\
& \leqslant \frac{\left(x^{p}-\mu_{1}^{p}\right)^{2}}{p\left(\mu_{2}^{p}-\mu_{1}^{p}\right)}\left(\int_{0}^{1} M_{p}^{-r}\left(x, \mu_{1} ; \omega\right) d \omega\right)^{\frac{1}{r}} \\
& \times\left(\left|\psi^{\prime}(x)\right|^{\mathrm{q}} \int_{0}^{1} \omega^{\mathrm{q}}[1-(s(1-\omega))] \mathrm{d} \omega+\left|\psi^{\prime}\left(\mu_{1}\right)\right|^{\mathrm{q}} \int_{0}^{1} \omega^{\mathrm{q}}[1-s \omega] \mathrm{d} \omega\right)^{\frac{1}{q}} \\
& +\frac{\left(\mu_{2}^{p}-x^{p}\right)^{2}}{p\left(\mu_{2}^{p}-\mu_{1}^{p}\right)}\left(\int_{0}^{1} M_{p}^{-r}\left(x, \mu_{2} ; \omega\right) d \omega\right)^{\frac{1}{r}} \\
& \times\left(\left|\psi^{\prime}(x)\right|^{q} \int_{0}^{1} \omega^{\mathrm{q}}[1-(s(1-\omega))] \mathrm{d} \omega+\left|\psi^{\prime}\left(\mu_{2}\right)\right|^{\mathrm{q}} \int_{0}^{1} \omega^{\mathrm{q}}[1-s \omega] \mathrm{d} \omega\right)^{\frac{1}{q}} \\
& \leqslant \frac{1}{p\left(\mu_{2}^{p}-\mu_{1}^{p}\right)} \times\left\{\left(x^{p}-\mu_{1}^{p}\right)^{2}\left(\frac{\beta(1,1)}{x^{r p-r}}{ }_{2} F_{1}\left(r-\frac{r}{p^{\prime}}, 1 ; 2 ; 1-\frac{\mu_{1}^{p}}{\chi^{p}}\right)\right)^{\frac{1}{r}}\right. \\
& \left(\left(\frac{1}{q+1}+s \beta(q+1,2)\right)\left|\psi^{\prime}(x)\right|^{q}+\left(\frac{1-s}{q+2}\right)\left|\psi^{\prime}\left(\mu_{1}\right)\right|^{q}\right)^{\frac{1}{q}} \\
& +\left(\mu_{2}^{\mathrm{p}}-\chi^{\mathrm{p}}\right)^{2}\left(\frac{\beta(1,1)}{\mu_{2}^{\mathrm{rp}-r}}{ }_{2} \mathrm{~F}_{1}\left(\mathrm{r}-\frac{\mathrm{r}}{\mathrm{p}}, 1 ; 2 ; 1-\frac{\chi^{\mathrm{p}}}{\mu_{2}^{\mathrm{p}}}\right)\right)^{\frac{1}{\mathrm{r}}} \\
& \left.\left(\left(\frac{1}{q+1}+s \beta(q+1,2)\right)\left|\psi^{\prime}(x)\right|^{q}+\left(\frac{1-s}{q+2}\right)\left|\psi^{\prime}\left(\mu_{2}\right)\right|^{q}\right)^{\frac{1}{q}}\right\} \text {, }
\end{aligned}
$$

which completes the proof.

Theorem 4.7. Let $\psi: \mathbb{A} \subseteq(0, \infty) \rightarrow \mathbb{R}$ be differential mapping on $\mathbb{A}^{\circ}$, where $\mu_{1}, \mu_{2} \in \mathbb{A}$ with $\mu_{1}<\mu_{2}, p \in \mathbb{R} \backslash\{0\}$ and $\psi^{\prime} \in \mathrm{L}\left[\mu_{1}, \mu_{2}\right]$. If $\left|\psi^{\prime}\right|^{\mathrm{q}}$ is s-type $\mathrm{p}$-convex for $\mathrm{s} \in[0,1]$ and $q \geqslant 1$, then $\forall x \in\left[\mu_{1}, \mu_{2}\right]$, we have

$$
\begin{aligned}
& \left|\psi(x)-\frac{p}{\mu_{2}^{p}-\mu_{1}^{p}} \int_{\mu_{1}}^{\mu_{2}} \frac{\psi(u)}{u^{1-p}} d u\right| \\
& \leqslant \frac{1}{p\left(\mu_{2}^{p}-\mu_{1}^{p}\right)} \times\left\{\left(x^{p}-\mu_{1}^{p}\right)^{2}\left(\frac{\beta(1,1)}{\mu_{1}^{r p-r}}{ }_{2} F_{1}\left(r-\frac{r}{p^{\prime}}, 1 ; 2 ; 1-\frac{x^{p}}{\mu_{1}^{p}}\right)\right)^{\frac{1}{r}}\right.
\end{aligned}
$$




$$
\begin{aligned}
& \times\left(\left(\frac{1}{q+1}+s \beta(q+1,2)\right)\left|\psi^{\prime}(x)\right|^{q}+\left(\frac{1-s}{q+2}\right)\left|\psi^{\prime}\left(\mu_{1}\right)\right|^{q}\right)^{\frac{1}{q}} \\
& +\left(\mu_{2}^{p}-x^{p}\right)^{2}\left(\frac{\beta(1,1)}{x^{r p-r}}{ }_{2} F_{1}\left(r-\frac{r}{p^{\prime}} 1 ; 2 ; 1-\frac{\mu_{2}^{p}}{x^{p}}\right)\right)^{\frac{1}{r}} \\
& \left.\left(\left(\frac{1}{q+1}+s \beta(q+1,2)\right)\left|\psi^{\prime}(x)\right|^{q}+\left(\frac{1-s}{q+2}\right)\left|\psi^{\prime}\left(\mu_{2}\right)\right|^{q}\right)^{\frac{1}{q}}\right\} .
\end{aligned}
$$

Proof. The proof is omitted as it is similar to the proof of Theorem 4.6. If we are choosing $p<0$, then as a result we get the inequality (4.3) is easily attained.

Theorem 4.8. Let $\psi: \mathbb{A} \subseteq(0, \infty) \rightarrow \mathbb{R}$ be differential mapping on $\mathbb{A}^{\circ}$, where $\mu_{1}, \mu_{2} \in \mathbb{A}$ with $\mu_{1}<\mu_{2}, p \in \mathbb{R} \backslash\{0\}$ and $\psi^{\prime} \in \mathrm{L}\left[\mu_{1}, \mu_{2}\right]$. If $\left|\psi^{\prime}\right|^{\mathrm{q}}$ is s-type $\mathrm{p}$-convex function for $\mathrm{s} \in[0,1]$, $\mathrm{p}>0, \mathrm{q} \geqslant 1$, then $\forall x \in\left[\mu_{1}, \mu_{2}\right]$, we have

$$
\begin{aligned}
& \left|\psi(x)-\frac{p}{\mu_{2}^{p}-\mu_{1}^{p}} \int_{\mu_{1}}^{\mu_{2}} \frac{\psi(u)}{u^{1-p}} d u\right| \\
& \leqslant \frac{\left(\frac{2-s}{2}\right)^{\frac{1}{q}}}{p\left(\mu_{2}^{p}-\mu_{1}^{p}\right)}\left\{( x ^ { p } - \mu _ { 1 } ^ { p } ) ^ { 2 } \left(\frac{\beta(1, r+1)}{x^{r p-r}}{ }_{2} F_{1}\left(r-\frac{r}{p^{\prime}}, 1 ; r+2 ; 1-\frac{\mu_{1}^{p}}{x^{p}}\right)^{\frac{1}{r}}\left(\left|\psi^{\prime}(x)\right|^{q}+\left|\psi^{\prime}\left(\mu_{1}\right)\right|^{q}\right)^{\frac{1}{q}}\right.\right. \\
& +\left(\mu_{2}^{p}-x^{p}\right)^{2}\left(\frac{\beta(r+1,1)}{\mu_{2}^{r p-r}}{ }_{2} F_{1}\left(r-\frac{r}{p^{\prime}}, r+1 ; r+2 ; 1-\frac{x^{p}}{\mu_{2}^{p}}\right)^{\frac{1}{r}}\left(\left|\psi^{\prime}(x)\right|^{q}+\left|\psi^{\prime}\left(\mu_{2}\right)\right|^{q}\right)^{\frac{1}{q}}\right\} .
\end{aligned}
$$

Proof. Applying Lemma 4.1, properties of modulus, Hölder's inequality and property of s-type $p$-convexity of $\left|\psi^{\prime}\right|^{q}$ and, we have

$$
\begin{aligned}
& \left|\psi(x)-\frac{p}{\mu_{2}^{p}-\mu_{1}^{p}} \int_{\mu_{1}}^{\mu_{2}} \frac{\psi(u)}{u^{1-p}} d u\right| \\
& =\frac{1}{p\left(\mu_{2}^{p}-\mu_{1}^{p}\right)}\left\{\left(x^{p}-\mu_{1}^{p}\right)^{2} \int_{0}^{1} M_{p}^{-1}\left(x, \mu_{1} ; \omega\right)(\omega)\left|\psi^{\prime}\left(M_{p}\left(x, \mu_{1} ; \omega\right)\right)\right| d \omega\right. \\
& +\left\{\left(\mu_{2}^{p}-x^{p}\right)^{2} \int_{0}^{1} M_{p}^{-1}\left(x, \mu_{2} ; \omega\right)(\omega)\left|\psi^{\prime}\left(M_{p}\left(x, \mu_{2} ; \omega\right)\right)\right| d \omega\right\} \\
& \leqslant \frac{\left(x^{p}-\mu_{1}^{p}\right)^{2}}{p\left(\mu_{2}^{p}-\mu_{1}^{p}\right)}\left(\int_{0}^{1} M_{p}^{-r}\left(x, \mu_{1} ; \omega\right) \omega^{r} d \omega\right)^{\frac{1}{r}}\left(\int_{0}^{1}\left|\psi^{\prime}\left(M_{p}\left(x, \mu_{1} ; \omega\right)\right)\right|^{q} d \omega\right)^{\frac{1}{q}} \\
& +\frac{\left(\mu_{2}^{p}-x^{p}\right)^{2}}{p\left(\mu_{2}^{p}-\mu_{1}^{p}\right)}\left(\int_{0}^{1} M_{p}^{-r}\left(x, \mu_{2} ; \omega\right) \omega^{r} d \omega\right)^{\frac{1}{r}}\left(\int_{0}^{1}\left|\psi^{\prime}\left(M_{p}\left(x, \mu_{2} ; \omega\right)\right)\right|^{q} d \omega\right)^{\frac{1}{q}}
\end{aligned}
$$




$$
\begin{aligned}
& \leqslant \frac{\left(x^{p}-\mu_{1}^{p}\right)^{2}}{p\left(\mu_{2}^{p}-\mu_{1}^{p}\right)}\left(\int_{0}^{1} M_{p}^{-r}\left(x, \mu_{1} ; \omega\right) \omega^{r} d \omega\right)^{\frac{1}{r}} \\
& \times\left(\int_{0}^{1}\left[[1-(s(1-\omega))]\left|\psi^{\prime}(x)\right|^{q}+[1-(s \omega)]\left|\psi^{\prime}\left(\mu_{1}\right)\right|^{q}\right] d \omega\right)^{\frac{1}{q}} \\
& +\frac{\left(\mu_{2}^{p}-x^{p}\right)^{2}}{p\left(\mu_{2}^{p}-\mu_{1}^{p}\right)}\left(\int_{0}^{1} M_{p}^{-r}\left(x, \mu_{2} ; \omega\right) \omega^{r} d \omega\right)^{\frac{1}{r}} \\
& \times\left(\int_{0}^{1}\left[[1-(s(1-\omega))]\left|\psi^{\prime}(x)\right|^{q}+[1-s \omega]\left|\psi^{\prime}\left(\mu_{2}\right)\right|^{q}\right] d \omega\right)^{\frac{1}{q}} \\
& \leqslant \frac{\left(x^{p}-\mu_{1}^{p}\right)^{2}}{p\left(\mu_{2}^{p}-\mu_{1}^{p}\right)}\left(\int_{0}^{1} M_{p}^{-r}\left(x, \mu_{1} ; \omega\right) \omega^{r} d \omega\right)^{\frac{1}{r}} \\
& \times\left(\left|\psi^{\prime}(x)\right|^{q} \int_{0}^{1}[1-(s(1-\omega))] d \omega+\left|\psi^{\prime}\left(\mu_{1}\right)\right|^{q} \int_{0}^{1}[1-s \omega] d \omega\right)^{\frac{1}{q}} \\
& +\frac{\left(\mu_{2}^{p}-x^{p}\right)^{2}}{p\left(\mu_{2}^{p}-\mu_{1}^{p}\right)}\left(\int_{0}^{1} M_{p}^{-r}\left(x, \mu_{2} ; \omega\right) \omega^{r} d \omega\right)^{\frac{1}{r}} \\
& \times\left(\left|\psi^{\prime}(x)\right|^{q} \int_{0}^{1}[1-(s(1-\omega))] d \omega+\left|\psi^{\prime}\left(\mu_{2}\right)\right|^{q} \int_{0}^{1}[1-s \omega] d \omega\right)^{\frac{1}{q}} \\
& \leqslant \frac{\left(\frac{2-s}{2}\right)^{\frac{1}{q}}}{p\left(\mu_{2}^{p}-\mu_{1}^{p}\right)}\left\{( x ^ { p } - \mu _ { 1 } ^ { p } ) ^ { 2 } \left(\frac{\beta(1, r+1)}{x^{r p}-r}{ }_{2} F_{1}\left(r-\frac{r}{p^{\prime}}, 1 ; r+2 ; 1-\frac{\mu_{1}^{p}}{\chi^{p}}\right)^{\frac{1}{r}}\left(\left|\psi^{\prime}(x)\right|^{q}+\left|\psi^{\prime}\left(\mu_{1}\right)\right|^{q}\right)^{\frac{1}{q}}\right.\right. \\
& +\left(\mu_{2}^{p}-x^{p}\right)^{2}\left(\frac{\beta(r+1,1)}{\mu_{2}^{r p-r}}{ }_{2} F_{1}\left(r-\frac{r}{p^{\prime}}, r+1 ; r+2 ; 1-\frac{x^{p}}{\mu_{2}^{p}}\right)^{\frac{1}{r}}\left(\left|\psi^{\prime}(x)\right|^{q}+\left|\psi^{\prime}\left(\mu_{2}\right)\right|^{q}\right)^{\frac{1}{q}}\right\}
\end{aligned}
$$

which completes the proof.

Theorem 4.9. Let $\psi: \mathbb{A} \subseteq(0, \infty) \rightarrow \mathbb{R}$ be differential mapping on $\mathbb{A}^{\circ}$, where $\mu_{1}, \mu_{2} \in \mathbb{A}$ with $\mu_{1}<\mu_{2}, p \in \mathbb{R} \backslash\{0\}$ and $\psi^{\prime} \in \mathrm{L}\left[\mu_{1}, \mu_{2}\right]$. If $\left|\psi^{\prime}\right|^{\mathrm{q}}$ is s-type $\mathrm{p}$-convex for $\mathrm{s} \in[0,1]$ and $\mathrm{q} \geqslant 1$, then $\forall x \in\left[\mu_{1}, \mu_{2}\right]$, we have

$$
\begin{aligned}
& \left|\psi(x)-\frac{p}{\mu_{2}^{p}-\mu_{1}^{p}} \int_{\mu_{1}}^{\mu_{2}} \frac{\psi(u)}{u^{1-p}} d u\right| \\
& \leqslant \frac{\left(\frac{2-s}{2}\right)^{\frac{1}{q}}}{p\left(\mu_{2}^{p}-\mu_{1}^{p}\right)}\left\{( x ^ { p } - \mu _ { 1 } ^ { p } ) ^ { 2 } \left(\frac{\beta(r+1,1)}{\mu_{1}^{r p}-r}{ }_{2} F_{1}\left(r-\frac{r}{p^{\prime}}, r+1 ; r+2 ; 1-\frac{x^{p}}{\mu_{1}^{p}}\right)^{\frac{1}{r}}\right.\right. \\
& \quad \times\left(\left|\psi^{\prime}(x)\right|^{q}+\left|\psi^{\prime}\left(\mu_{1}\right)\right|^{q}\right)^{\frac{1}{q}} \\
& +\left(\mu_{2}^{p}-x^{p}\right)^{2}\left(\frac{\beta(1, r+1)}{x^{r p-r}}{ }_{2} F_{1}\left(r-\frac{r}{p}, 1 ; r+2 ; 1-\frac{\mu_{2}^{p}}{x^{p}}\right)^{\frac{1}{r}}\left(\left|\psi^{\prime}(x)\right|^{q}+\left|\psi^{\prime}\left(\mu_{2}\right)\right|^{q}\right)^{\frac{1}{q}}\right\} .
\end{aligned}
$$

Proof. The proof is omitted as it is similar to the proof of Theorem 4.8. If we are choosing $p<0$, then as a result we get the inequality (4.4) is easily attained. 
Theorem 4.10. Let $\psi: \mathbb{A} \subseteq(0, \infty) \rightarrow \mathbb{R}$ be differential mapping on $\mathbb{A}^{\circ}$, where $\mu_{1}, \mu_{2} \in \mathbb{A}$ with $\mu_{1}<\mu_{2}, p \in \mathbb{R} \backslash\{0\}$ and $\psi^{\prime} \in \mathrm{L}\left[\mu_{1}, \mu_{2}\right]$. If $\left|\psi^{\prime}\right|^{\mathrm{q}}$ is s-type $\mathrm{p}$-convex for $\mathrm{q} \geqslant 1, \mathrm{p}>0$ and $s \in[0,1]$, then $\forall x \in\left[\mu_{1}, \mu_{2}\right]$, we have

$$
\begin{aligned}
& \mid \psi(x)- \frac{p}{\mu_{2}^{p}-\mu_{1}^{p}} \int_{\mu_{1}}^{\mu_{2}} \frac{\psi(u)}{u^{1-p}} d u \mid \\
& \leqslant \frac{1}{p\left(\mu_{2}^{p}-\mu_{1}^{p}\right)}\left(\frac{1}{r+1}\right)^{\frac{1}{r}}\left\{\left(x^{p}-\mu_{1}^{p}\right)^{2}\left(\Delta_{9}\left|\psi^{\prime}(x)\right|^{q}+\Delta_{10}\left|\psi^{\prime}\left(\mu_{1}\right)\right|^{q}\right)^{\frac{1}{q}}\right. \\
&\left.\quad+\left(\mu_{2}^{p}-x^{p}\right)^{2}\left(\Delta_{11}\left|\psi^{\prime}(x)\right|^{q}+\Delta_{12}\left|\psi^{\prime}\left(\mu_{2}\right)\right|^{q}\right)^{\frac{1}{q}}\right\},
\end{aligned}
$$

where

$$
\begin{aligned}
& \Delta_{9}=\frac{\beta(1,1)}{\chi^{q p}-q}{ }_{2} F_{1}\left(q-\frac{q}{p}, 1 ; 2 ; 1-\frac{\mu_{1}^{p}}{x^{p}}\right)-s \frac{\beta(2,1)}{\chi^{q p}-q}{ }_{2} F_{1}\left(q-\frac{q}{p}, 2 ; 3 ; 1-\frac{\mu_{1}^{p}}{\chi^{p}}\right), \\
& \Delta_{10}=\frac{\beta(1,1)}{\chi^{q p}-q}{ }_{2} F_{1}\left(q-\frac{q}{p}, 1 ; 2 ; 1-\frac{\mu_{1}^{p}}{\chi^{p}}\right)-s \frac{\beta(1,2)}{\chi^{q p}-q}{ }_{2} F_{1}\left(q-\frac{q}{p}, 1 ; 3 ; 1-\frac{\mu_{1}^{p}}{\chi^{p}}\right), \\
& \Delta_{11}=\frac{\beta(1,1)}{\mu_{2}^{q p-q}}{ }_{2} F_{1}\left(q-\frac{q}{p^{2}}, 1 ; 2 ; 1-\frac{x^{p}}{\mu_{2}^{p}}\right)-s \frac{\beta(1,2)}{\mu_{2}^{q p}-q}{ }_{2} F_{1}\left(q-\frac{q}{p}, 1 ; 3 ; 1-\frac{x^{p}}{\mu_{2}^{p}}\right) \text {, } \\
& \Delta_{12}=\frac{\beta(1,1)}{\mu_{2}^{q p-q}}{ }_{2} F_{1}\left(q-\frac{q}{p^{2}}, 1 ; 2 ; 1-\frac{\mu_{2}^{p}}{\chi^{p}}\right)-s \frac{\beta(2,1)}{\mu_{2}^{q p}-q}{ }_{2} F_{1}\left(q-\frac{q}{p^{2}}, 2 ; 3 ; 1-\frac{\chi^{p}}{\mu_{2}^{p}}\right) .
\end{aligned}
$$

Proof. Applying Lemma 4.1, properties of modulus, Hölder's inequality, and the property of s-type $p$-convexity of $\left|\psi^{\prime}\right|^{q}$, we have

$$
\begin{aligned}
& \left|\psi(x)-\frac{p}{\mu_{2}^{p}-\mu_{1}^{p}} \int_{\mu_{1}}^{\mu_{2}} \frac{\psi(u)}{u^{1-p}} d u\right| \\
& =\frac{1}{p\left(\mu_{2}^{p}-\mu_{1}^{p}\right)}\left\{\left(x^{p}-\mu_{1}^{p}\right)^{2} \int_{0}^{1} M_{p}^{-1}\left(x, \mu_{1} ; \omega\right)(\omega)\left|\psi^{\prime}\left(M_{p}\left(x, \mu_{1} ; \omega\right)\right)\right| d \omega\right. \\
& +\left\{\left(\mu_{2}^{p}-x^{p}\right)^{2} \int_{0}^{1} M_{p}^{-1}\left(x, \mu_{2} ; \omega\right) \omega\left|\psi^{\prime}\left(M_{p}\left(x, \mu_{2} ; \omega\right)\right)\right| d \omega\right\} \\
& \leqslant \frac{\left(x^{p}-\mu_{1}^{p}\right)^{2}}{p\left(\mu_{2}^{p}-\mu_{1}^{p}\right)}\left(\int_{0}^{1} \omega^{r} d \omega\right)^{\frac{1}{r}}\left(\int_{0}^{1} M_{p}^{-q}\left(x, \mu_{1} ; \omega\right)\left|\psi^{\prime}\left(M_{p}\left(x, \mu_{1} ; \omega\right)\right)\right|^{q} d \omega\right)^{\frac{1}{q}} \\
& +\frac{\left(\mu_{2}^{p}-x^{p}\right)^{2}}{p\left(\mu_{2}^{p}-\mu_{1}^{p}\right)}\left(\int_{0}^{1} \omega^{r} d \omega\right)^{\frac{1}{r}}\left(\int_{0}^{1} M_{p}^{-q}\left(x, \mu_{2} ; \omega\right)\left|\psi^{\prime}\left(M_{p}\left(x, \mu_{2} ; \omega\right)\right)\right|^{q} d \omega\right)^{\frac{1}{q}}
\end{aligned}
$$




$$
\begin{aligned}
& \leqslant \frac{\left(x^{p}-\mu_{1}^{p}\right)^{2}}{p\left(\mu_{2}^{p}-\mu_{1}^{p}\right)}\left(\int_{0}^{1} \omega^{r} d \omega\right)^{\frac{1}{r}} \\
& \times\left(\int_{0}^{1} M_{p}^{-q}\left(x, \mu_{1} ; \omega\right)\left[[1-(s(1-\omega))]\left|\psi^{\prime}(x)\right|^{q}+[1-s \omega]\left|\psi^{\prime}\left(\mu_{1}\right)\right|^{q}\right] d \omega\right)^{\frac{1}{q}} \\
& +\frac{\left(\mu_{2}^{p}-x^{p}\right)^{2}}{p\left(\mu_{2}^{p}-\mu_{1}^{p}\right)}\left(\int_{0}^{1} \omega^{r} d \omega\right)^{\frac{1}{r}} \\
& \times\left(\int_{0}^{1} M_{p}^{-q}\left(x, \mu_{2} ; \omega\right)\left[[1-(s(1-\omega))]\left|\psi^{\prime}(x)\right|^{q}+[1-s \omega]\left|\psi^{\prime}\left(\mu_{2}\right)\right|^{q}\right] d \omega\right)^{\frac{1}{q}} \\
& \leqslant \frac{\left(x^{p}-\mu_{1}^{p}\right)^{2}}{p\left(\mu_{2}^{p}-\mu_{1}^{p}\right)}\left(\int_{0}^{1} \omega^{r} d \omega\right)^{\frac{1}{r}}\left(\left|\psi^{\prime}(x)\right|^{q} \int_{0}^{1} M_{p}^{-q}\left(x, \mu_{1} ; \omega\right)[1-(s(1-\omega))] d \omega\right. \\
& \left.+\left|\psi^{\prime}\left(\mu_{1}\right)\right|^{q} \int_{0}^{1} M_{p}^{-q}\left(x, \mu_{1} ; \omega\right)[1-s \omega] d \omega\right)^{\frac{1}{q}} \\
& +\frac{\left(\mu_{2}^{p}-x^{p}\right)^{2}}{p\left(\mu_{2}^{p}-\mu_{1}^{p}\right)}\left(\int_{0}^{1} \omega^{r} d \omega\right)^{\frac{1}{r}} \times\left(\left|\psi^{\prime}(x)\right|^{q} \int_{0}^{1} M_{p}^{-q}\left(x, \mu_{2} ; \omega\right)[1-(s(1-\omega))] d \omega\right. \\
& \left.+\left|\psi^{\prime}\left(\mu_{2}\right)\right|^{q} \int_{0}^{1} M_{p}^{-q}\left(x, \mu_{2} ; \omega\right)[1-s \omega] d \omega\right)^{\frac{1}{q}} \\
& \leqslant \frac{1}{p\left(\mu_{2}^{p}-\mu_{1}^{p}\right)}\left(\frac{1}{r+1}\right)^{\frac{1}{r}} \times\left\{\left(x^{p}-\mu_{1}^{p}\right)^{2}\left(\Delta_{9}\left|\psi^{\prime}(x)\right|^{q}+\Delta_{10}\left|\psi^{\prime}\left(\mu_{1}\right)\right|^{q}\right)^{\frac{1}{q}}\right. \\
& \left.+\left(\mu_{2}^{p}-x^{p}\right)^{2}\left(\Delta_{11}\left|\psi^{\prime}(x)\right|^{q}+\Delta_{12}\left|\psi^{\prime}\left(\mu_{2}\right)\right|^{q}\right)^{\frac{1}{q}}\right\} .
\end{aligned}
$$

This is the required proof.

Theorem 4.11. Let $\psi: \mathbb{A} \subseteq(0, \infty) \rightarrow \mathbb{R}$ be differential mapping on $\mathbb{A}^{\circ}$, where $\mu_{1}, \mu_{2} \in \mathbb{A}$ with $\mu_{1}<\mu_{2}, p \in \mathbb{R} \backslash\{0\}$ and $\psi^{\prime} \in \mathrm{L}\left[\mu_{1}, \mu_{2}\right]$. If $\left|\psi^{\prime}\right|^{\mathrm{q}}$ is s-type $p$-convex for $\mathrm{s} \in[0,1]$ and $q \geqslant 1$, then $\forall x \in\left[\mu_{1}, \mu_{2}\right]$, we have

$$
\begin{aligned}
& \left|\psi(x)-\frac{p}{\mu_{2}^{p}-\mu_{1}^{p}} \int_{\mu_{1}}^{\mu_{2}} \frac{\psi(u)}{u^{1-p}} d u\right| \\
& \leqslant \frac{1}{p\left(\mu_{2}^{p}-\mu_{1}^{p}\right)}\left(\frac{1}{r+1}\right)^{\frac{1}{r}}\left\{\left(x^{p}-\mu_{1}^{p}\right)^{2}\left(\Delta_{9}^{\prime}\left|\psi^{\prime}(x)\right|^{q}+\Delta_{10}^{\prime}\left|\psi^{\prime}\left(\mu_{1}\right)\right|^{q}\right)^{\frac{1}{q}}\right. \\
& \left.+\left(\mu_{2}^{p}-x^{p}\right)^{2}\left(\Delta_{11}^{\prime}\left|\psi^{\prime}(x)\right|^{q}+\Delta_{12}^{\prime}\left|\psi^{\prime}\left(\mu_{2}\right)\right|^{q}\right)^{\frac{1}{q}}\right\},
\end{aligned}
$$

where

$$
\begin{aligned}
& \Delta_{9}^{\prime}=\frac{\beta(1,1)}{\mu_{1}^{q p-q}}{ }_{2} F_{1}\left(q-\frac{q}{p}, 1 ; 2 ; 1-\frac{x^{p}}{\mu_{1}^{p}}\right)-s \frac{\beta(1,2)}{\mu_{1}^{p-1}}{ }_{2} F_{1}\left(q-\frac{q}{p^{2}}, 1 ; 3 ; 1-\frac{x^{p}}{\mu_{1}^{p}}\right), \\
& \Delta_{10}^{\prime}=\frac{\beta(1,1)}{\mu_{1}^{q p-q}}{ }_{2} F_{1}\left(q-\frac{q}{p}, 1 ; 2 ; 1-\frac{x^{p}}{\mu_{1}^{p}}\right)-s \frac{\beta(2,1)}{\mu_{1}^{p-1}}{ }_{2} F_{1}\left(q-\frac{q}{p}, 2 ; 3 ; 1-\frac{x^{p}}{\mu_{1}^{p}}\right),
\end{aligned}
$$




$$
\begin{aligned}
& \Delta_{11}^{\prime}=\frac{\beta(1,1)}{\chi^{q p}-q}{ }_{2} F_{1}\left(q-\frac{q}{p^{2}}, 1 ; 2 ; 1-\frac{\mu_{2}^{p}}{\chi^{p}}\right)-s \frac{\beta(2,1)}{\chi^{p-1}}{ }_{2} F_{1}\left(q-\frac{q}{p^{2}}, 2 ; 3 ; 1-\frac{\mu_{2}^{p}}{\chi^{p}}\right), \\
& \Delta_{12}^{\prime}=\frac{\beta(1,1)}{\chi^{q p}-q}{ }_{2} F_{1}\left(q-\frac{q}{p^{p}}, 1 ; 2 ; 1-\frac{\mu_{2}^{p}}{\chi^{p}}\right)-s \frac{\beta(1,2)}{\chi^{p-1}}{ }_{2} F_{1}\left(q-\frac{q}{p^{p}}, 1 ; 3 ; 1-\frac{\mu_{2}^{p}}{\chi^{p}}\right) .
\end{aligned}
$$

Proof. The rest of proof is similar to the proof of Theorem 4.10. If we are choosing $p<0$, then as a result we get the inequality (4.6) is easily attained.

\section{Conclusion}

We have inferred few new refinements of Ostrowski type inequality utilizing a new class of convexity namely s-type $p$-convex function. We have also deduced some new special cases of the newly established results also. We showed that this class of functions had some nice properties, which other convex functions had as well. We proved that our new class of $s$-type $p$-convex function is very larger with respect to the known class of functions, like convex and harmonically convex. New versions of Ostrowski type inequalities for the differentiable functions are obtained. This shows that our results are very interesting and unique. It will be an intriguing idea for the researchers for future research to consider the results acquired in this paper on different directions like fractional calculus, quantum calculus, time scale calculus, on coordinates with help of various kinds of convex functions.

\section{References}

[1] Khan MA, Chu YM, Khan TU and Khan J (2017). "Some new inequalities of Hermite-Hadamard type for s-convex functions with applications". Open Math. 15: 1414-1430. http://doi.org/10.20852/ntmsci. 2016318838

[2] Özdemir ME, Yildiz C, Akdemir AO and Set E (2013). “On some inequalities for s-convex functions and applications". J. Ineq. Appl. 333: 2-11.

[3] Özcan S and İşcan İ (2019). "Some new Hermite-Hadamard type inequalities for s-convex functions and their applications". J. Inequal. Appl. 201: 1-11.

[4] Xi BY and Qi F (2012). "Some integral inequalities of Hermite-Hadamard type for convex functions with applications to means". J. Funct. Spaces. Appl. Article ID 980438. 1-14. http://doi.org/10.1155/2012/ 980438

[5] Hardy GH, Little JE and Polya G (1952). "Inequalities". Cambridge, UK. Cambridge University Press. cambridge mathematical library.

[6] Butt SI, Tariq M, Aslam A, Ahmad H and Nofel TA (2021), "Hermite-Hadamard type inequalities via generalized harmonic exponential convexity". J. Funct. Spaces. 1-12. http://doi .org/10.1155/2021/5533491

[7] Butt SI, Kashuri A, Tariq M, Nasir J, Aslam A and Geo W (2020). "Hermite-Hadamard-type inequalities via n-polynomial exponential-type convexity and their applications". Adv. Differ. Equ. 508. http://doi.org/ 10.1186/s13662-020-02967-5

[8] Butt SI, Kashuri A, Tariq M, Nasir J, Aslam A and Geo W (2020). "n-polynomial exponential-type pconvex function with some related inequalities and their applications". Heliyon. http://doi.org/10.1016/ j.heliyon. 2020.e05420

[9] Ostrowski A (1938). "Über die Absolutabweichung einer differentiebaren funktion von ihren integralmittelwert". Comment. Math. Helv. 10: 226-227.

[10] Alomari M, Darus M, Dragomir SS and Cerone P (2010). “Ostrowski type inequalities for functions whose derivatives are s-convex in the second sense". Applied Mathematics Letters. 23(9). 1071-1076.

[11] Dragomir SS (1998). "On the Ostrowski's integral inequality for mappings with bounded variation and applications". Math. Ineq. Appl. 1(2). 
[12] Alomari M and Darus M (2010). "Some Ostrowski type inequalities for quasi-convex functions with applications to special means". RGMIA Res. Rep. Coll, 13(2).

[13] Set E, Sarikaya MZ and Özdemir ME (2010). "Some Ostrowski's Type Inequalities for Functions whose Second Derivatives are s-Convex in the Second Sense and Applications". arXiv preprint arXiv:1006.2488.

[14] Pachpatte BG (2000). "On an inequality of Ostrowski type in three independent variables". J. Math. Anal. Appl. 249: 583-591. http://doi.org/10.1006/jmaa.2000.6913

[15] Niculescu CP and Persson LE (2006). "Convex functions and their applications". Springer, New York.

[16] İşcan İ (2016). Hermite-Hadamard type inequalities for p-convex functions, I. J. Anal. Appl. 11(2): 137-145.

[17] Rashid S, İşcan İ, Baleanu D and Chu YM (2020). "Generation of new fractional inequalities via npolynomials s-type convexity with applications". Adv. Differ. Equ. 264.

[18] Sommerfeld A (1949). "Cylinder and Sphere Problems". Partial Differential Equations in Physics.

[19] Assche WV (2006). "Ordinary Special Functions". Encyclopedia of Mathematical Physics.

[20] Abramowitz M and Stegun IA (1965). "Handbook of Mathematical Functions with Formulas". Graphs, and Mathematical Tables. Dover, New York.

[21] İşcan İ(2014). "Hermite-Hadamard type inequalities for harmonically convex functions". Hacet. J. Math. Stat. 43(6): 935-942.

[22] İşcan İ (2016). "Ostrowski type inequalities for p-convex functions". New Trends in Mathematical Sciences. 3: $140-150$. 